ARTICLE

\title{
High efficiency blue organic light-emitting diodes with below-bandgap electroluminescence
}

\author{
Maria Vasilopoulou ${ }^{1 凶}$, Abd. Rashid bin Mohd Yusoff (10 ${ }^{2 凶}$, Matyas Daboczi (10 ${ }^{3}$, Julio Conforto ${ }^{4}$, \\ Anderson Emanuel Ximim Gavim4, Wilson Jose da Silva4, Andreia Gerniski Macedo4, Anastasia Soultati', \\ George Pistolis ${ }^{1}$, Fabio Kurt Schneider (iD ${ }^{4}$, Yifan Dong ${ }^{5}$, Polina Jacoutot ${ }^{5}$, Georgios Rotas ${ }^{6}$, Jin Jang (i) ${ }^{7}$, \\ Georgios C. Vougioukalakis ${ }^{6}$, Christos L. Chochos (iD ${ }^{8 凶}$, Ji-Seon Kim (i) ${ }^{3}$ \& Nicola Gasparini (i) ${ }^{5 凶}$
}

Blue organic light-emitting diodes require high triplet interlayer materials, which induce large energetic barriers at the interfaces resulting in high device voltages and reduced efficiencies. Here, we alleviate this issue by designing a low triplet energy hole transporting interlayer with high mobility, combined with an interface exciplex that confines excitons at the emissive layer/electron transporting material interface. As a result, blue thermally activated delay fluorescent organic light-emitting diodes with a below-bandgap turn-on voltage of $2.5 \mathrm{~V}$ and an external quantum efficiency (EQE) of $41.2 \%$ were successfully fabricated. These devices also showed suppressed efficiency roll-off maintaining an EQE of $34.8 \%$ at $1000 \mathrm{~cd} \mathrm{~m}^{-2}$. Our approach paves the way for further progress through exploring alternative device engineering approaches instead of only focusing on the demanding synthesis of organic compounds with complex structures.

\footnotetext{
${ }^{1}$ Institute of Nanoscience and Nanotechnology, National Centre for Scientific Research Demokritos, Terma Patriarchou Grigoriou, Agia Paraskevi, Greece. ${ }^{2}$ Department of Chemical Engineering, Pohang University of Science and Technology (POSTECH), Pohang, Gyeongbuk, Republic of Korea. ${ }^{3}$ Department of Physics and Centre for Processable Electronics, Imperial College London, London, UK. ${ }^{4}$ Universidade Tecnologica Federal do Parana, GPGEl, Curitiba, Parana, Brazil. ${ }^{5}$ Department of Chemistry and Centre for Processable Electronics, Imperial College London, London, UK. ${ }^{6}$ Department of Chemistry, National and Kapodistrian University of Athens, Athens, Greece. ${ }^{7}$ Advanced Display Research Center, Department of Information Display, Kyung Hee University, Dongdaemoon-gu, Seoul, South Korea. ${ }^{8}$ Institute of Chemical Biology, National Hellenic Research Foundation, Athens, Greece. ${ }^{凶}$ email: m.vasilopoulou@inn. demokritos.gr; abdrashid@postech.ac.kr; chochos@eie.gr; n.gasparini@imperial.ac.uk
} 
O rganic light-emitting diodes (OLEDs) with blue emission of high efficiency and operational stability are very important in solid-state lighting, information storage of high density, and vivid displays ${ }^{1-5}$. The low-efficiency issue of purely fluorescent devices was successfully addressed by the development of noble metal-based organometallic phosphors that enabled the fabrication of blue OLEDs with external quantum efficiencies (EQEs) exceeding $30 \%^{6-9}$. However, the high cost and limited reserve of noble metal phosphors have been the driving force for developing purely organic thermally activated delay fluorescent (TADF) emitters relying on radiative deactivation from singlet states with $100 \%$ quantum efficiency ${ }^{10-13}$. Through careful design and compositional engineering of such emitters $^{14-17}$, the EQEs of blue and sky-blue TADF OLEDs have been steadily improved from $19.5 \%$ in $2014^{18}$ to $38.2 \%$ in $2019^{19}$ and further to $38.4 \%$ in $2021^{20}$, hence surpassing in performance the best blue phosphorescence OLEDs ${ }^{9}$. These devices to successfully confine both charges and excitons within the blue emitter, which is generally dispersed into an appropriate matrix to form the emissive layer (EML), require high lowest unoccupied molecular orbital (LUMO) and triplet energy $\left(\mathrm{E}_{\mathrm{T}}\right)$ interlayers ${ }^{21}$. As a result, large energetic barriers are present at the interfaces resulting in high device voltages, reduced efficiencies, and high efficiency roll-off due to the carrier accumulation at these interfaces.

In a standard OLED architecture, the electrical carrier injection takes place via the highest occupied molecular orbital (HOMO) and the LUMO of the hole injection layer (HIL) and electron injection interlayer (EIL) injection interlayer, respectively. To reduce the charge injection barriers, it is necessary to adjust the energy levels of the HIL/EIL with the work function $\left(\mathrm{W}_{\mathrm{F}}\right)$ of the respective electrode. However, to confine excitons (both singlet and triplet) within the blue EML, interlayer materials with high singlet energy and $\mathrm{E}_{\mathrm{T}}$ (commonly above $3.0 \mathrm{eV}^{22}$ ) are necessary. These requirements are met in blue OLEDs by fabricating multilayer stacks, including separate charge injection, transporting, and exciton-confining interlayers. These stacks include several interfaces where charges and excitons are accumulated, deteriorating the device performance and causing a drop of EQE in high current densities $(J)$ known as efficiency roll-off ${ }^{23-25}$. One way to prevent these parasitic effects is, therefore, the design and fabrication of simplified structures ${ }^{26-28}$.

Here we apply a device-engineering approach that allowed us to demonstrate blue TADF OLEDs with high figure of merits, including below bandgap turn-on voltage of $2.5 \mathrm{~V}$ and the highest reported to date EQE for any blue OLED of $41.2 \%$. The high OLED performance was made possible through the application of a low $\mathrm{E}_{\mathrm{T}}(2.14 \mathrm{eV})$ hole transport material $(\mathrm{HTM})$, rationally designed with a high hole mobility of $7.5 \times 10^{-3} \mathrm{~cm}^{2}(\mathrm{~V} \mathrm{~s})^{-1}$. This HTM served as a hole "reservoir" influencing both hole and electron carrier injection, and transport and subsequent exciton formation at the EML/electron transport material (ETM) interface. Effective exciton confinement due to the formation of an interface exciplex between the ambipolar host and the ETM prevents exciton diffusion towards the low $\mathrm{E}_{\mathrm{T}} \mathrm{HTM}$, resulting in minimal energy loss, below-bandgap electroluminescence (EL), high efficiency, improved lifetime, and reduced efficiency roll-off.

\section{Results}

Materials and device conceptual design. Besides technical specifications, any developed material for OLED applications needs to be able to scale up to hundreds of kilograms, at an acceptable cost and environmental impact for real-world applications. Thiophene-quinoxaline (TQ) copolymers are considered very promising organic photovoltaic donor materials of low synthetic complexity and easy scale-up at multigram quantities ${ }^{29}$. In this work, we synthesized two fluorinated-TQ copolymers, abbreviated as T2fQ and TQ2f, containing two fluorine (F) atoms in thiophene and quinoxaline, respectively. The synthetic procedure is shown in Fig. 1a. The chemical structure of each copolymer is shown in the upper panel of Fig. 1b. The identity, purity,
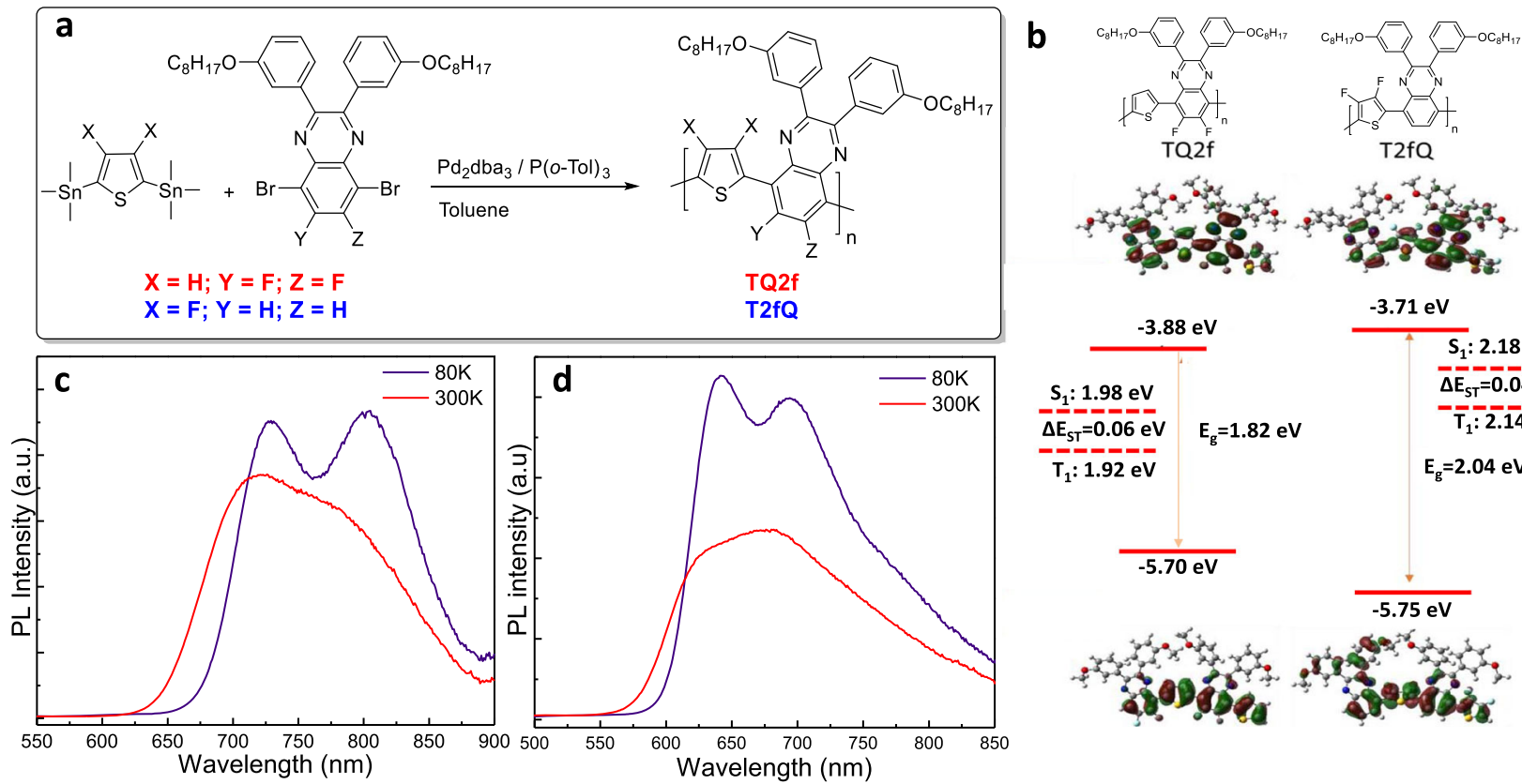

Fig. 1 Properties of the hole-transporting interlayers. a The synthetic procedure for the preparation of fluorinated TQs. $\mathbf{b}$ The chemical structures of TQ2f and $\mathrm{T} 2 \mathrm{fQ}$ used as hole-transporting interlayers, the calculated frontier orbitals and energy levels as derived from cyclic voltammetry, and absorption measurements and the lower excited singlet and triplet levels of TQ2f and T2fQ. The room temperature (RT, $300 \mathrm{~K}$ ) and low temperature ( $80 \mathrm{~K}$ ) photoluminescence spectra of $\mathbf{c} T Q 2 f$ and $\mathbf{d} T 2 \mathrm{fQ}$ thin films. 
molecular weight, and optoelectronic properties of both compounds were ascertained using ${ }^{1} \mathrm{H}$ and ${ }^{13} \mathrm{C}$ nuclear magnetic resonance $\left({ }^{1} \mathrm{H}-\mathrm{NMR}\right.$ and ${ }^{13} \mathrm{C}-\mathrm{NMR}$, respectively), gel permeation chromatography (GPC), absorption and emission spectroscopy, and cyclic voltammetry during oxidation (Supplementary Figs. 14 and Supplementary Tables 1 and 2). The latter enabled the estimation of the fluorinated TQ's HOMO, whereas absorption measurements defined the optical bandgap value of each copolymer. The calculated frontier orbitals and the corresponding energy levels (Supplementary Note 1) are presented in Fig. 1b. The replacement of the $\mathrm{F}$ atoms from the quinoxaline to the thiophene has significant effect on the energy levels along with the dihedral angle between the two adjustments building blocks (Supplementary Fig. 5). This is expected to largely affect the intramolecular charge transfer and molecular packing, and, consequently, the carrier mobility of these copolymers in the solid state $^{30-32}$. Photoluminescence (PL) spectra and transient PL (TRPL) decay curves measured either in ambient at a wide temperature range from $80 \mathrm{~K}$ to room temperature $(300 \mathrm{~K}, \mathrm{RT})$ (Fig. 1c, d and Supplementary Figs. 6 and 7) or in oxygen/ nitrogen environments (at RT) (Supplementary Figs. 8 and 9) enabled the estimation of the lowest excited singlet $\left(\mathrm{S}_{1}\right)$ and triplet $\left(\mathrm{T}_{1}\right)$ states (Fig. $1 \mathrm{~b}$ ), while also indicating that these HTMs are RT phosphorescence materials. From the energy levels point of view, these materials are far from being ideal interlayers for direct contact with the EML, where requirements for high LUMO and high $\mathrm{E}_{\mathrm{T}}$ to avoid electron leakage and prevent exciton quenching are very strict.

Contrarily to conventional OLED architectures where excitons are directly formed in the emitter, OLEDs using donor:acceptor (D:A) species interacting in the excited state, the so-called exciplexes, have gained much attention after the pioneering work of Adachi and colleagues ${ }^{17,33}$. Analogously to TADF emitters, exciplexes display an innately small energy separation $\left(\Delta \mathrm{E}_{\mathrm{ST}}\right)$ between their charge-transfer excited states (these are either singlet, ${ }^{1} \mathrm{CT}$, or triplet, ${ }^{3} \mathrm{CT}$, in character), indicating a feasible way toward theoretical $100 \%$ exciton harvesting ${ }^{17}$, when applied either as hosts or as emitters ${ }^{33}$. As an alternative strategy, the use of interface exciplexes to strongly localize excitons and improve OLED efficiency has also been reported ${ }^{22}$, albeit there are stringent requirements that should be fulfilled for optimum device performance, especially for blue OLEDs ${ }^{34}$. In particular, ${ }^{1} \mathrm{CT} /$ ${ }^{3} \mathrm{CT}$ states have to be lower than the local excitation energies $\left({ }^{1} \mathrm{LE} /\right.$ ${ }^{3} \mathrm{LE}$ ) of both donor and acceptor constituents for the successful confinement of all excitons within the exciplex. At the same time, ${ }^{1} \mathrm{CT} /{ }^{3} \mathrm{CT}$ states must be higher than the emitter's LE states in order to succeed efficient energy transfer towards the latter at RT and avoid back-electron transfer towards the donor or acceptor constituents. In blue OLEDs, in particular interface exciplexes with high ${ }^{1} \mathrm{CT} /{ }^{3} \mathrm{CT}$ states lying above those of the blue emitters $\left(\sim 3.0 \mathrm{eV}^{9}\right)$ are required, which necessitates the selection of large bandgap donor and acceptor constituents. We rationally selected an ambipolar host, namely bis(diphenylphosphine oxide)dibenzofuran (DBFPO) ${ }^{35}$ (Supplementary Note 2 and Supplementary Figs. 10-12), which served as the donor constituent combined with an ETM, namely diphenyl[4-(triphenylsilyl)phenyl]phosphine oxide $(\mathrm{TSPO} 1)^{36}$, to serve as the acceptor constituent of the interface exciplex. DBFPO bears diplenylphosphine oxide acceptor and dibenzofuran donor groups; TSPO1 consists of triplenyl silyl and triphenyl oxide acceptor groups. The multiple $\pi-\sigma$ interactions through C-Si direct coupling substantially lower its HOMO energy, rendering this compound an excellent conventional acceptor ${ }^{37}$.

We investigated the possible exciplex formation between TSPO1 and DBFPO constituents by monitoring the absorption and emission spectra of the monomers in solution and of pristine and mixed films. Supplementary Fig. 13 presents the absorption and emission spectra of TSPO1 and DBFPO monomers in dilute solution $\left(5 \times 10^{-7} \mathrm{M}\right)$ and films. The PL spectrum of DBFPO film exhibits a red-shifted broad shoulder, which is attributed to excimer emission (in accordance to ref. ${ }^{35}$ ). By subtracting the PL spectrum of the monomer, we found that this emission is centered at $361 \mathrm{~nm}$, which is in well agreement with previous reports ${ }^{35}$. We next investigated the absorption and emission properties of mixed (in molecular ratio of 10:1, 1:1, and 1:10) TSPO1 and DBFPO films. As depicted in Supplementary Fig. 14, their absorption spectra derive from the superposition of the constituents, which indicates that these materials do not interact in the ground state. Differently, the PL spectra of the mixed films exhibit strict differences; we note that these spectra were taken using a $330 \mathrm{~nm}$ short-pass filter to cut most of the ultraviolet (UV) emission of the pristine materials and focus on the longer wavelengths where exciplex formation-if any-is expected. In the spectra taken in ambient conditions at RT (Fig. 2a), in addition to the contribution of the monomers and DBFPO's excimer emission, which appears as a shoulder at $\sim 361 \mathrm{~nm}$ in the $1: 1$ ratio and mostly in the $1: 10$ film with the higher DBFPO content, a new distinct peak appears at longer wavelengths (centered at $440-450 \mathrm{~nm}$ ). This peak exhibits higher intensity in the spectrum of 1:1 mixed film, where larger interaction between the two constituents is expected. Notably, this peak becomes even more pronounced in the spectra taken in nitrogen (Fig. 2b) and at low temperatures (Fig. 2c). The Gaussian peak deconvolution (Supplementary Fig. 15) revealed that the PL spectrum of the 1:1 mixed film can be considered as the superposition of narrow peaks derived from the monomers, one broad peak centered at $370 \mathrm{~nm}$, which is related to the DBFPO's excimer, and one broad peak centered at $440 \mathrm{~nm}$. The latter is due to excited state interaction between TSPO1 and DBFPO, and can be attributed to exciplex formation between the constituents. This is in agreement with the higher intensity of this peak observed in the 1:1 film compared to the 1:10 and 10:1 films in air, as well as the increased intensity of the peak in nitrogen and at low temperature in the 1:1 mixture. The broad peak at $\sim 440 \mathrm{~nm}$ is therefore attributed to charge-transfer emission from the complex excited dimer.

The PL studies of pristine compounds and mixed films revealed the exciplex formation between the two constituents. The higher energy peak in the PL spectrum of the mixed films is the remaining emission of the constituents due to incomplete energy transfer between them due to low energy difference $(\Delta \mathrm{E})$ between their LUMOs $(0.2 \mathrm{eV}$, Fig. $2 \mathrm{~d})$. A $\Delta E>0.5 \mathrm{eV}$ is needed to allow $100 \%$ charge transfer between the consituents ${ }^{38}$. However, even in this case the formation of an interface exciplex might be beneficial for the OLED operation, as it is expected to confine excitons within the EML/ETM interface. These excitons can then be transferred to the emitter's molecules instead of being diffused towards the HTM/EML interface where excitons are probably quenched. Our studies excluded the possibility that the PL broadening in the $1: 1$ mixed film is due to different orientation/conformation of the constituent molecules in the mixed film. Supplementary Fig. 16 and Fig. 2c present the evolution of PL spectra of TSPO1 and DBFPO pristine and their mixtures films upon increasing temperature. In pristine TSPO1 and TSPO1:DBFPO 10:1 films, the phosphorescence of the ETM is clearly observed at low temperature in the wavelength range of interest (around 400-550 $\mathrm{nm}$ ). Upon heating to reach RT, TSPO1 exhibits no distinct emission, whereas the 10:1 film presents a small but distinct shoulder at $440 \mathrm{~nm}$. Differently, the DBFPO and 1:10 films present a broad red-shifted shoulder (reaching up to $600 \mathrm{~nm}$ ) in both films, especially in pristine DBFPO. This could be due to molecular rearrangement upon heating or the induction of photochemical processes from continuous 

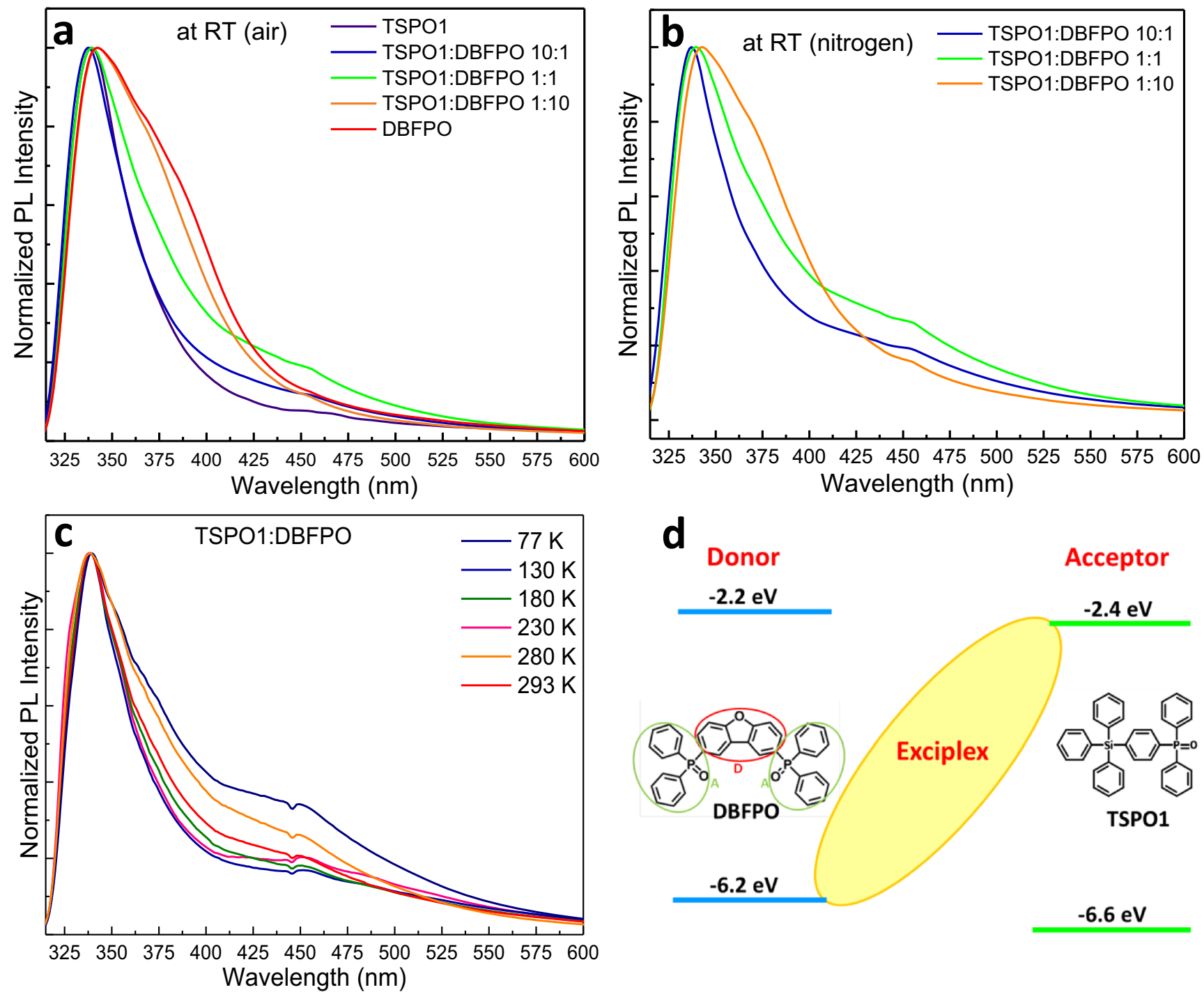

$-6.6 \mathrm{eV}$

Fig. 2 Illustration of the interface exciplex formation. a Room temperature (RT) normalized PL spectra of TSPO1 and DBFPO pristine films and mixtures. b The PL spectra of mixtures taken at RT in nitrogen. c Temperature-dependent PL spectra of TSPO1:DBFPO 1:1 mixed film. $\mathbf{d}$ The chemical structures and frontier orbitals of DBFPO and TSPO1, and the illustration of interfacial exciplex formation.

excitation. A similar behavior was previously observed for DBFPO upon UV exposure in air $^{35}$, which was also verified by our experiments (Supplementary Fig. 17a). This can be attributed to significant molecular rearrangement of this material upon heating/exposure and/or due to photochemical processes ${ }^{35}$. Contrarily to pristine DBFPO, negligible change was observed in the TSPO1:DBFPO 1:1 upon UV irradiation (Supplementary Fig. 17b), which represents a clear indication of strong molecular interactions between the excited states of the constituents in the mixed film. This supports the exciplex formation between different molecules rather than the interactions/rearrangements of similar molecules.

Peformances of blue TADF OLEDs. To test our conceptual device-engineering approach, we next fabricated blue OLEDs based on the 5-(2,12-di-tert-butyl-5,9-dioxa-13b-boranaphtho [3,2,1-de] anthracen-7-yl)-10,15-diphenyl-10,15-dihydro-5Hdiindolo $\left[3,2-\mathrm{a}: 3^{\prime}, 2^{\prime}-\mathrm{c}\right]$ carbazole (TDBA-DI) TADF emitter that previously enabled the fabrication of blue OLEDs with an EQE of $38.2 \%{ }^{19}$. Our devices consisted of the following: indium tin oxide (ITO) anode, N,N'-Di(1-naphthyl)-N, $\mathrm{N}^{\prime}$-diphenyl-(1,1'-biphenyl)-4, $4^{\prime}$-diamine (a-NPD) hole injection layer (HIL), fluorinated TQ or 3,5-di(9H-carbazol-9-yl)-N,N-diphenylaniline (DCDPA) HTM $^{19}$, 20\% TDBA-DI doped in DBFPO host, TSPO1 ETM, and LiF/Al cathode. All device layers were vacuum evaporated, except for TQ derivatives, which were processed from tetrahydrofuran (THF) solution in order not to dissolve the NPD underneath. The solution concentration and processing conditions were optimized to obtain smooth films and maximum device performance (Supplementary Fig. 18). Figure 3a presents the frontier orbitals of organic materials and $\mathrm{W}_{\mathrm{F}}$ values of metal contacts, considering energy vacuum level alignment before contact. It indicates that TQs are inappropriate for use as HTM in direct contact with the EML, as they could facilitate electron leakage and exciton quenching at the HTM/EML interface. Despite the negative prevision, the T2fQ device exhibited blue emission with color coordinates of $(0.15,0.28)$ (Fig. $3 b)$, peak EL at $458 \mathrm{~nm}$ (Fig. 3c), and ultra-low turn-on voltage of $2.5 \mathrm{~V}$ (Fig. 3d), a current efficiency of $72.2 \mathrm{~cd} \mathrm{~A}^{-1}$, and a power efficiency (PE) of $87.2 \mathrm{~lm} \mathrm{~W}^{-1}$ (Fig. 3e and Table 1). The EL spectrum was identical with the PL one (Supplementary Fig. 19a), which suggests that T2fQ and DBFPO:TSPO1 interface exciplex have no contribution to the emitted wavelengths. The low device turn-on voltage corresponds to below-bandgap EL. The origin of 

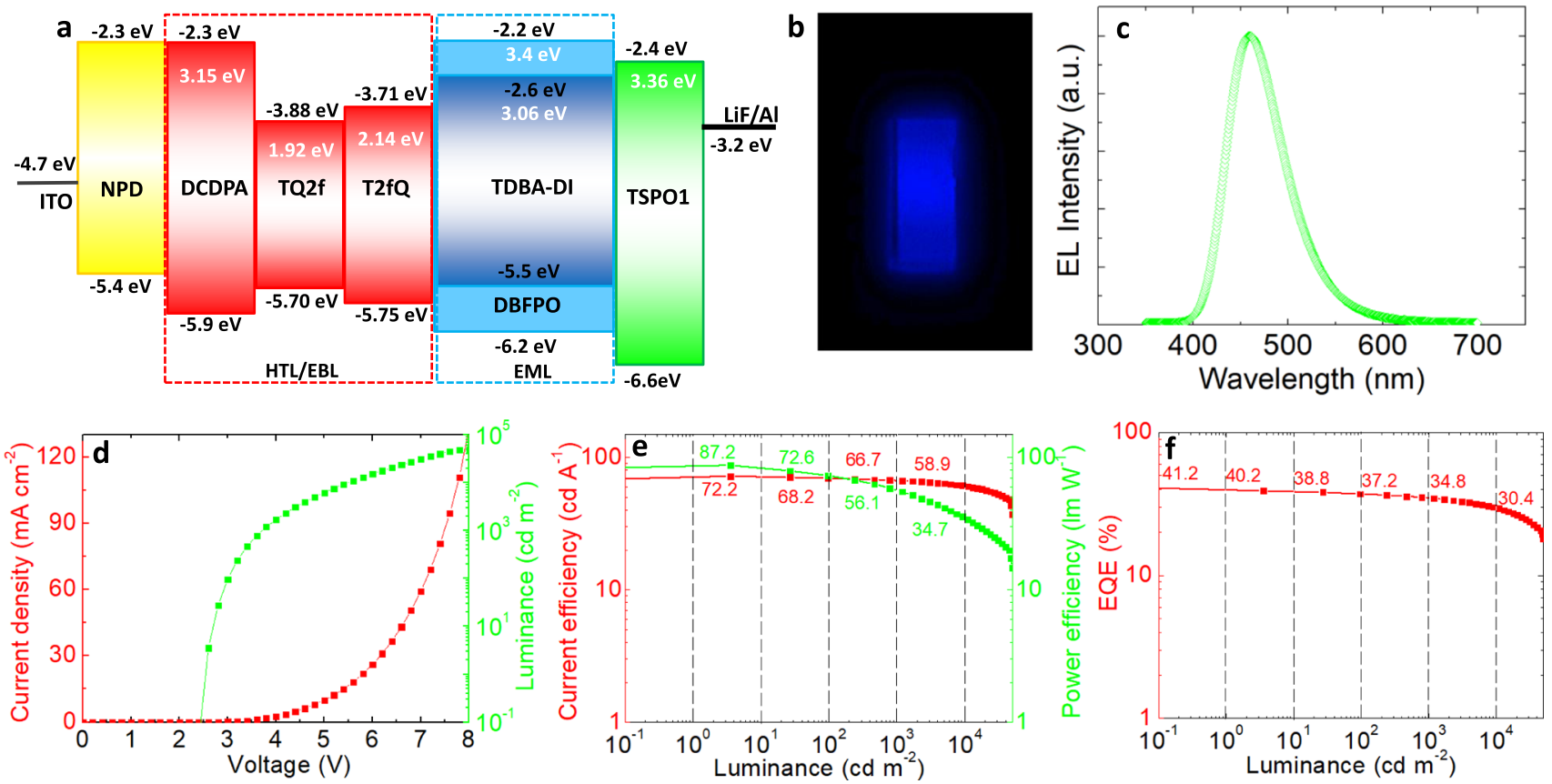

Fig. 3 Performance of optimized blue TADF OLEDs and efficiency roll-off. a The energy-level diagram of the layers sequence in the blue TADF OLED device architecture considering vacuum level alignment before contact. Besides HOMO and LUMO energies of organic layers, the triplet energy of the emitter, host, and interlayer materials are also presented. b Photograph of a working OLED pixel with an active area of $25 \mathrm{~mm}^{2}$ and $\mathbf{c}$ electroluminescence spectrum of the same device. $\mathbf{d}$ Current density-luminance-voltage characteristics, e current and power efficiency vs. luminance, and $\mathbf{f} E Q E$ vs. luminance characteristics for the OLED.

Table 1 Summary of the performance characteristics of our best-performing blue OLEDs and comparison with the bestperforming, to date, blue TADF OLEDs ${ }^{19,20}$ and blue PHOLED?.

\begin{tabular}{|c|c|c|c|c|c|c|c|c|c|}
\hline HTM//ETM & $E M L / \lambda_{\text {em }}(\mathrm{nm})$ & $\begin{array}{l}\text { Turn-on } \\
\text { voltage }(\mathrm{V})^{\mathrm{a}}\end{array}$ & $\begin{array}{l}\text { FWHM } \\
(\mathbf{n m})^{\mathbf{b}}\end{array}$ & $\begin{array}{l}\text { Maximum } \\
\text { luminance } \\
\left(\mathbf{c d ~ m}^{-2}\right)\end{array}$ & \multicolumn{3}{|c|}{ Maximum efficiency } & CIE $1931(x, y)^{c}$ & Ref. \\
\hline DCDPA//TSPO1 & $\begin{array}{l}\text { DBFPO : TDBA- } \\
\mathrm{DI} / 458\end{array}$ & 2.5 & 65 & 37590 & $\begin{array}{l}68.0 \\
(48.0)\end{array}$ & $\begin{array}{l}82.1 \\
(26.3)\end{array}$ & $\begin{array}{l}37.8 \\
(29.0)\end{array}$ & $(0.15,0.28)$ & This work \\
\hline T2fQ//TSPO1 & $\begin{array}{l}\text { DBFPO : TDBA- } \\
\text { DI/458 }\end{array}$ & 2.5 & 65 & 49530 & $\begin{array}{l}72.2 \\
(61.7)\end{array}$ & $\begin{array}{l}87.2 \\
(36.8)\end{array}$ & $\begin{array}{l}41.2 \\
(34.6)\end{array}$ & $(0.15,0.28)$ & This work \\
\hline $\begin{array}{l}\text { DCDPA// } \\
\text { DBFPO/TPBi }\end{array}$ & $\begin{array}{l}\text { DBFPO : TDBA- } \\
\text { DI/458 }\end{array}$ & 3.1 & 65 & 47680 & $\begin{array}{l}64.4 \\
(57.0)\end{array}$ & $\begin{array}{l}57.2 \\
(33.0)\end{array}$ & $\begin{array}{l}38.2 \\
(34.3)\end{array}$ & $(0.15,0.28)$ & Ref. ${ }^{19}$ \\
\hline${ }^{d} \mathrm{mCP} / / \mathrm{TmPyPB}$ & $\begin{array}{l}\text { CbBPCb: } \\
\text { Flrpic/473 }\end{array}$ & - & 75 & 7000 & 53.6 & 50.6 & 30 & - & Ref. ${ }^{9}$ \\
\hline \multicolumn{10}{|c|}{$\begin{array}{l}\text { CE current efficiency, } P E \text { power efficiency. } \\
\text { aTurn-on voltage at } 1 \mathrm{~cd} \mathrm{~m}^{-2} \text {. } \\
\text { bFWHM at } 10 \mathrm{~mA} \mathrm{~cm}^{-2} \text {. } \\
\text { cCIE } 1931 \text { coordinates. } \\
\text { dBest phosphorescence OLED. }\end{array}$} \\
\hline
\end{tabular}

such emission has been previously attributed to CT excitons present at the EML/ETM interface under sub-bandgap driving conditions $^{39}$. The interface exciplex having CT states lying well below the emitter's LE states (the $440 \mathrm{~nm}$ peak emission of the interface exciplex corresponds to CT energy of $2.8 \mathrm{~nm}$, which is below the emitter's LUMO, Fig. 3a), could contribute to the below-bandgap EL and explain the low turn-on voltage. Moreover, our device achieved a world record EQE of 41.2\% (Fig. 3f), with a mean EQE of 34.6\% (Supplementary Fig. 19b). Remarkably, a very low-efficiency roll-off of $8.6 \%$ at $1000 \mathrm{~cd} \mathrm{~m}^{-2}$ and $21.6 \%$ at $5000 \mathrm{~cd} \mathrm{~m}^{-2}$ was also obtained. Notably, the performance of the reference devices using the high-energy LUMO/high $\mathrm{E}_{\mathrm{T}}$ DCDPA was slightly inferior to that of the T2fQ OLEDs
(Supplementary Fig. 20). These devices achieved an EQE of $37.6 \%$, which is very close to the efficiency of $38.2 \%$ exhibited by the previously reported OLEDs using DCDBA/TDBA-DI:DBFPO interfaces (Table 1) ${ }^{19}$. Notably, they still exhibited a belowbandgap turn-on voltage of $2.5 \mathrm{~V}$ (compared to $3.1 \mathrm{~V}$ of ref. ${ }^{19}$ ), resulting in a higher PE of $82.1 \mathrm{~lm} \mathrm{~W}^{-1}$ (compared to $57.2 \mathrm{~lm}$ $\mathrm{W}^{-1}$ of ref. ${ }^{19}$ ). However, the device using TQ2f was far inferior with an EQE hardly reaching 11.75\% (Supplementary Fig. 21), an indication that the mobility of the HTM plays a crucial role in the device performance.

To shed light to OLED performance, we next tried to determine the emitting dipole orientation within the EML of the champion OLEDs by performing angle-resolved PL 

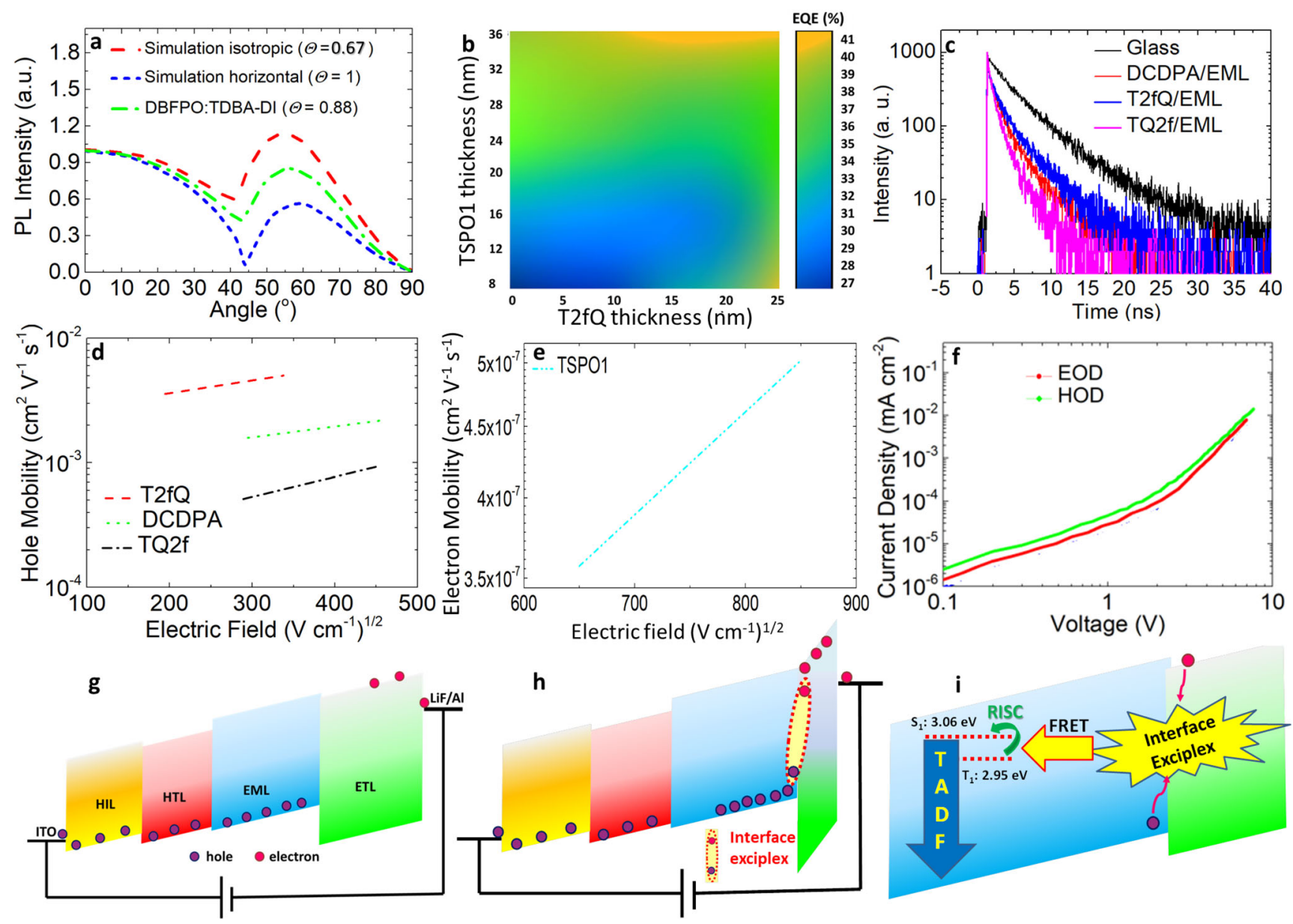

h
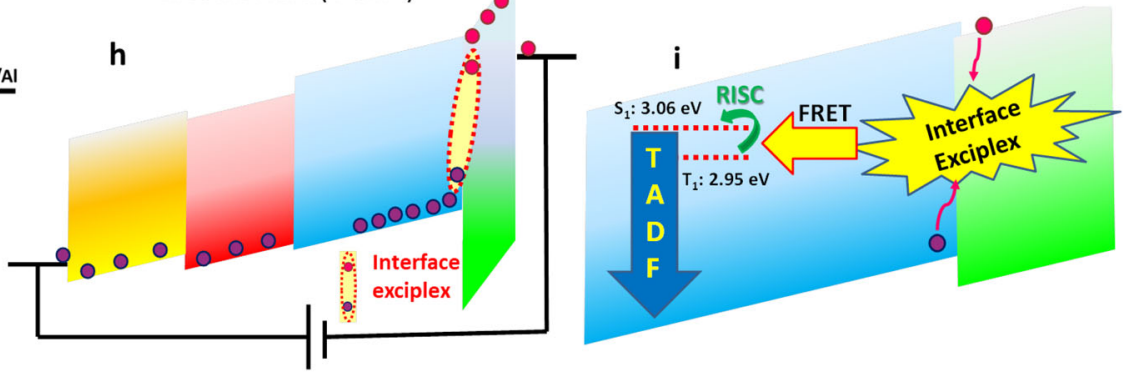

Fig. 4 Proposed operation mechanism. a Dependence of polarized PL intensity on the emission angle and $\mathbf{b}$ simulations for the thickness optimization of TSPO1 and T2fQ ETM and HTM. c TRPL decay curves of the EML on glass, DCPPA, T2fQ, and TQ2f films. d Variation of hole mobility vs. the applied field for the three different HTMs used in this study. e Variation of electron mobility on the applied field of the TSPO1 ETM. $\mathbf{f}$ Current densities measured in hole-only (ITO/NPD/TQ2f/DBFPO 20\%-TDBA-DI/NPD/AI) and electron-only (ITO/TSPO1/DBFPO 20\%-TDBA-DI/TSPO1/LiF/AI) devices. Schematic response of an ITO/NPD/T2fQ/EML/ETL/LiF/AI device under forward applied bias. $\mathbf{g}$ The electric field (slope of the energy levels) is evenly distributed across the device. Holes and electrons are injected from the anode and cathode contact, respectively, and transported through the HTL and ETL towards the EML. Due to the large difference in hole and electron mobilities of the HTM and ETM, respectively, holes are reaching the EML before electrons are transported within the ETL. h, i Holes reaching and start building up at the EML/ETL interface screen the electric field to the ETL. The redistribution of the electric field into the ETL accelerates electron injection/transport and electrons reach the EML/ETL interface where they subsequently form interfacial exciplexes with holes in the DBFPO layer. Interface exciplex confines excitons and transfers them to the emitter.

measurements in a $30 \mathrm{~nm}$ film consisting of TDBA-DI $20 \%$ doped in DBFPO. Figure 4a includes polarized PL measurements and optical simulations for isotropic oriented $\left(\Theta_{/ /}=0.67\right)$ and horizontally oriented $\left(\Theta_{/ /}=1\right)$ emitters. In general, horizontal emitting dipole $\left(\Theta_{/ /}=1\right)$ show stronger light emission at the front direction, hence improving outcoupling efficiency ${ }^{40,41}$. The results shown in Fig. 4a confirm that when the EML is deposited on T2fQ, the larger portion of the emitting dipoles is horizontally oriented $\left(\Theta_{/ /}=0.88\right)$. This is in good agreement with ref. ${ }^{19}$, indicating that $\mathrm{T} 2 \mathrm{fQ}$ underlayer preserves the same horizontal orientation of the emitting dipoles in EML as the previously applied DCDPA does. This result, however, cannot explain the best performance of the T2fQ-based devices compared to DCDPA ones, even though careful adjustment of HTM and ETM thicknesses enabled the achievement of the highest possible EQE (Fig. 4b). Aiming to reveal the origin of such remarkable device performance, we also measured the PL quantum yields in 20\% doped TDBA-DI:DBFPO films deposited on DCDPA, TQ2f, and T2fQ layers; the corresponding values were quite close, in particular $0.88,0.83$ and 0.92 , respectively. Furthermore, TRPL measurements were taken on the same TDBA-DI:DBFPO films deposited on the different HTMs and on glass substrate (Fig. 4c and Supplementary Table 3). The results indicated comparable degree of exciton confinement in both EML/T2fQ and EML/ DCDPA bilayers. Hole and electron mobilities of the HTM and ETM interlayers were further estimated with time-of-flight (TOF) measurements (Fig. 4d, e). It is seen that fluorine substitution in thiophene (T2fQ) instead of quinoxaline (TQ2f) highly improves the hole mobility of T2fQ to nearly $7.5 \times 10^{-3} \mathrm{~cm}^{2}(\mathrm{~V} \mathrm{~s})^{-1}$, which is fivefold higher relative to that of DCDPA and one order of magnitude above that of TQ2f. The electron mobility measured for TSPO1 was nearly four orders of magnitude lower, in accordance to the results published by others ${ }^{41}$. As a result, highly unbalanced hole and electron currents are expected, which, however, is not the case for T2fQ-based devices where nearly identical electron and hole currents were obtained in electrononly and hole-only (HOD) devices, respectively (Fig. 4f). On the contrary, highly unbalanced hole/electron currents were measured in the T2fQ-based HODs (Supplementary Fig. 22), which normally deteriorates the device performance. 
Based on our findings to explain the highly balanced transport and superior performance of T2fQ-based OLEDs, we propose a device operation mechanism that involves the following: (i) highly efficient hole transport through T2fQ upon the application of an external bias, (ii) effective blocking of holes by the deep HOMO of TSPO1, (iii) electric field redistribution across TSPO1 and acceleration of electron transport therein due to built-up of holes at the EML/TSPO1 interface, (iv) exciton formation at the EML/TSPO1 interface and confinement by the interface exciplex, and (v) exciton transfer to the emitter and emission of blue light. This concept is well supported by the obtained data and is illustrated in Fig. 4g-i, which shows the electric fields (in the term of energy bands bending) in an ITO/NPD/T2fQ/DBFPO:TDBA$\mathrm{DI} / \mathrm{TSPO} 1 / \mathrm{LiF} / \mathrm{Al}$ device upon the application of forward bias and the different steps of device mechanism proposed above. Exciton confinement by the interface exciplex prohibits their diffusion across the $30 \mathrm{~nm}$-thick EML, where a high probability of reaching the low energy levels of $\mathrm{T} 2 \mathrm{fQ}$ exists, especially for the triplet excitons having diffusion lengths in the range of 15-30 $\mathrm{nm}^{42,43}$. Upon the formation of an interface exciplex, even though the intermolecular charge transfer between donor: acceptor and acceptor constituents is not as high as $100 \%$, effective Förster resonant energy transfer results in excitons being transferred to the blue emitter (Fig. 4i).

To further support interface exciplex formation, we fabricated OLEDs with low emitter concentration doping in the EML. These devices exhibited a pronounced blue shift in their EL emission upon decreasing the emitter concentration (Supplementary Fig. 23). This is due to the exciplex emission and indicates suppressed energy transfer towards the emitter at lower doping levels. To unambiguously demonstrate the interface exciplex originated high-performance enhancement in our OLEDs, we inserted a $10 \mathrm{~nm}$-thick DBFPO to serve as a buffer layer between the EML and TSPO1. By decreasing the concentration doping of the TDBA-DI emitter in the DBFPO host, besides a moderate decrease in EL intensity, attributed to suppressed energy transfer from DBFPO host to the emitter, non-profound EL shift was evident. Moreover, a significant reduction in the device performance was obtained through increasing the DBFPO buffer layer thickness from 0 to $10 \mathrm{~nm}$ (Supplementary Table 4), clearly demonstrating the critical role of interface exciplex formed between DBFPO and TSPO1 constituents in the device performance. In addition, the exciplex emission is clearly seen in the EL spectra taken at $2.5 \mathrm{~V}$ compared to those taken at $3.0 \mathrm{~V}$ forward bias (Supplementary Fig. 24). This indicates that the below-bandgap EL is due to CT excitons from interface exciplex, in accordance with previous studies ${ }^{39}$.

OLED lifetime measurements. Besides high efficiencies and low turn-on voltage, the operational lifetime of an OLED is crucial for practical applications. Significant improvement in half-lifetime (T50) (up to $14 \mathrm{~h}$ for initial luminance of $1000 \mathrm{~cd} \mathrm{~m}^{-2}$ ) was obtained in our OLEDs, using not only T2fQ but also DCDPA and TQ2f interlayers, compared to the recently published OLEDs with the same emitter (i.e., $2 \mathrm{~h})^{19}$ (Fig. 5a and Supplementary Fig. 25), indicating decreased charge carrier accumulation at the DBFPO/TSPO1 interface, which would be the origin of severe degradation ${ }^{44}$. The formation of the interface exciplex, however, strongly confines and rapidly transfers excitons towards the emitter. The excited host molecule instability-induced degradation, which was recently proposed by Adachi and colleagues ${ }^{44}$ as a major cause of emission loss in TADF OLEDs, is largely alleviated in our devices. Moreover, a minor change in the driving voltage was obtained even for a high luminance of $5000 \mathrm{~cd} \mathrm{~m}^{-2}$ (Fig. 5a), thus protecting our device from Joule heating and subsequent degradation ${ }^{45}$. The shape of the degraded EL spectra was in all cases similar to the initial ones (Fig. 5b and Supplementary Figs. 26 and 27), indicating that the degradation of those devices does not involve any changes in the recombination zone. This is in agreement with our proposed mechanism, which involves strong confinement of excitons within the DBFPO/ TSPO1 interface and direct transfer towards the emitter ${ }^{46}$ Moreover, TOF secondary ion mass spectrometry (TOF-SIMS) measurements taken in the optimized OLED structures before and after degradation (Fig. 5c, d) revealed well-defined interfaces in the profiles, indicating strong resistance of the selected materials in degradation factors ${ }^{47}$. However, a large tail of the ITO profile extended within the whole device and a significant reduction of its intensity at the interface with NPD after degradation suggest that the primary degradation mechanism in these OLEDs derives through decomposition and diffusion of ITO electrode within the device, which has also been considered responsible for many types of OLED degradation ${ }^{48,49}$.

\section{Discussion}

We fabricated blue TADF OLEDs using a low $\mathrm{E}_{\mathrm{T}}$ HTM, a fluorinated TQ copolymer, which was designed with a high hole mobility of $7.5 \times 10^{-3} \mathrm{~cm}^{2}(\mathrm{~V} \mathrm{~s})^{-1}$. We combined this material with an interface exciplex formed between an ambipolar host and an ETM at the cathode device side. The HTM not only facilitated fast hole transfer towards the EML but also improved electron injection upon electric field redistribution within the low-mobility ETM. Interface exciplex strongly confined the generated excitons at the host/ETM interface, thus preventing leakage towards the exciton quenching HTM. These excitons were directly transferred to the blue emitter, thus suppressing charge and energy losses. Below-bandgap EL, highly improved efficiencies, suppressed efficiency roll-off and prolonged device lifetime were obtained, thus realizing the potential of using alternative interlayer materials and device architectures instead of well-designed emitters for further advancements in solution-processed LEDs.

\section{Methods}

Synthesis of organic materials. Hole injection material NPD (98\% purity) and ETM TSPO1 (>99\% purity) were purchased from Lumtec and Ossila, respectively, and were used as received. TDBA-DI and DCDPA were synthesized according to ref. ${ }^{19}$. DBFPO was synthesized using a modified previously published procedure (see Supplementary Note 2). The syntheses of 5,8-dibromo-2,3-bis(3-(octyloxy) phenyl)quinoxaline,5,8-dibromo-6,7-difluoro-2,3-bis(3-(octyloxy)phenyl) quinoxaline and bis(trimethylstannyl)thiophene were performed according to already published procedures ${ }^{50}$. Then, (3,4-difluorothiophene-2,5-diyl)bis(trimethylstannane) was purchased from Derthon Optoelectronic Materials Science Technology Co., Ltd. The experimental condition for the polymerizations is analytically described below: the 5,8-dibromo-2,3-bis(3-(octyloxy)phenyl)quinoxaline ( 0.5 $\mathrm{mmol})$ was combined with (3,4-difluorothiophene-2,5-diyl)bis(trimethylstannane) (0.5 mmol) for the T2fQ and the 5,8-dibromo-6,7-difluoro-2,3-bis(3-(octyloxy) phenyl)quinoxaline $(0.5 \mathrm{mmol})$ was combined with the bis(trimethylstannyl)thiophene $(0.5 \mathrm{mmol})$ for the TQ2f. Then, distilled toluene $(0.025 \mathrm{M})$ was added at the reaction mixtures. Finally, tris(dibenzylideneacetone)dipalladium $(0)\left[\mathrm{Pd}_{2}\left(\mathrm{dba}_{3}\right)\right]$ ( 0.02 equiv. $)$ and tri(o-tolyl $)$ phosphine $\left[\mathrm{P}(\mathrm{o}-\text { tol })_{3}\right](0.08$ equiv. $)$ were added, and the reaction mixture was stirred at $120^{\circ} \mathrm{C}$ under argon atmosphere for $24 \mathrm{~h}$. The polymers were purified by precipitation in methanol, filtered, and washed using a Soxhlet apparatus with methanol, acetone, hexane, and chloroform. The chloroform fractions were evaporated under reduced pressure and the polymers were precipitated in methanol, filtered through $0.45 \mathrm{~mm}$ PTFE filter and finally dried under high vacuum, rendering a dark brown solid with metallic appearance. The yields of the resulting polymers are the following: $\mathrm{T} 2 \mathrm{fQ}=93 \%$ and $\mathrm{TQ} 2 \mathrm{f}=91 \%$. All reactions are air and light sensitive, and, therefore, were performed under argon and in the dark. All glasswares were washed using detergent (Teepol), rinsed with excess water, acetone, and methylene dichloride, and dried in an oven at $120^{\circ} \mathrm{C}$. All solvents and reagents were purchased from Aldrich. Toluene was distilled using calcium hydride $\left(\mathrm{CaH}_{2}\right)$ and benzophenone prior to polymerization.

Device fabrication and measurement of EL characteristics. The device structure was as follows: ITO/NPD (40 nm)/DCDPA or TQ2f or T2fQ (15 nm)/DBFPO 20\% doped with TDBA-DI $(30 \mathrm{~nm}) / \mathrm{TSPO} 1(30 \mathrm{~nm}) / \mathrm{LiF}(1 \mathrm{~nm}) / \mathrm{Al}(100 \mathrm{~nm})$. Prior to an 

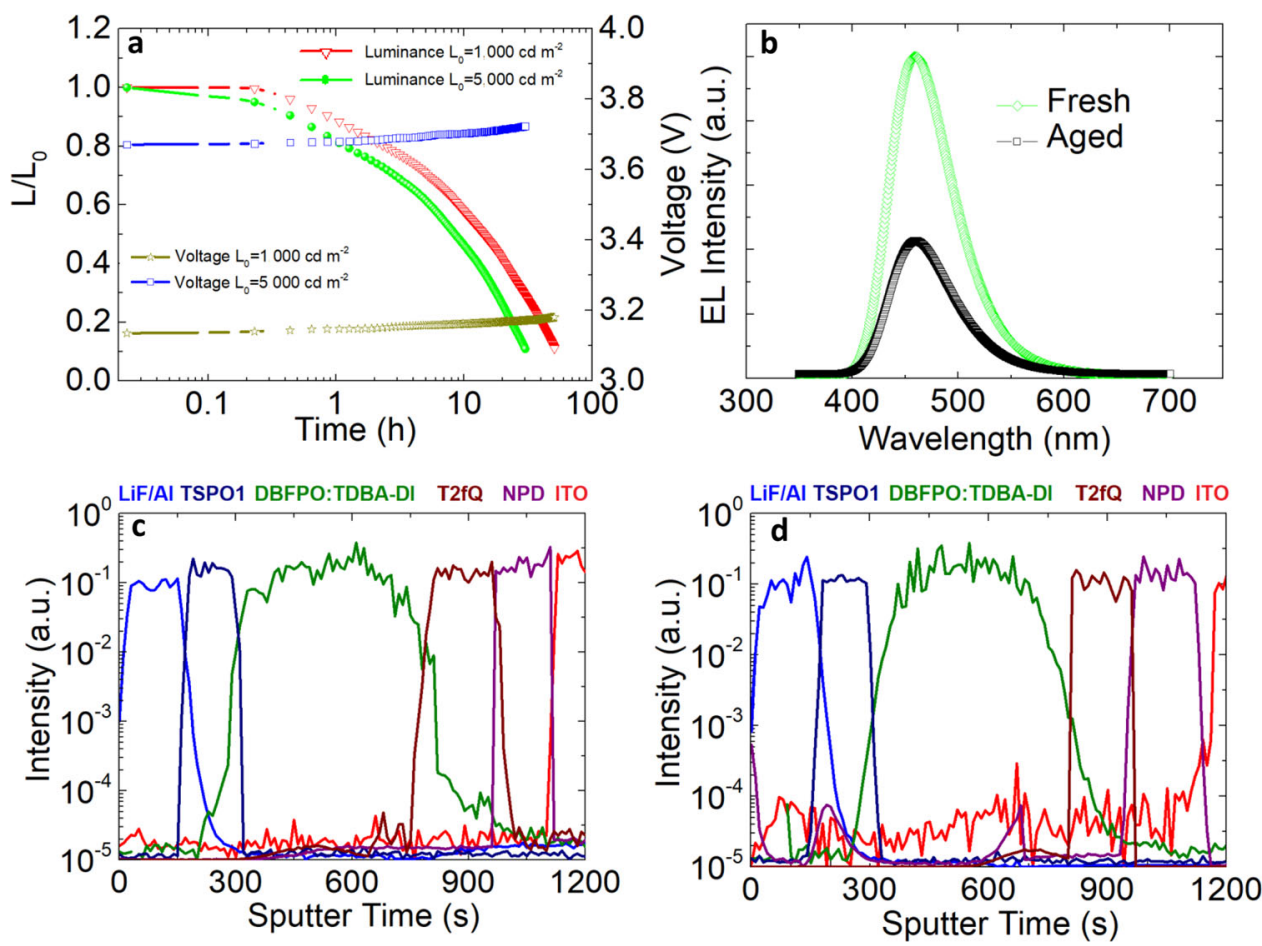

Fig. 5 Lifetime measurements. a Variation of normalized luminance vs. time and driving voltage vs. time for initial luminance of 1000 or $5000 \mathrm{~cd} \mathrm{~m}^{-2}$. b Electroluminescence spectra of the fresh and degraded device. TOF-SIMS profiles of the $\mathbf{c}$ fresh and $\mathbf{d}$ degraded OLED stack: the $x$-axis represents the materials depth distribution starting with the cathode interface from data point 0 and reaching to the ITO anode electrode.

OLED fabrication, cleaned glass substrates were patterned with a $250 \mathrm{~nm}$-thick ITO anode deposited through a shadow mask using RF magnetron sputtering in an inert argon environment at a pressure of $1 \times 10^{-6} \mathrm{mbar}$ at a rate of $0.06 \AA \mathrm{s}^{-1}$. The substrates were heated to $\sim 250^{\circ} \mathrm{C}$ during deposition to increase the ITO conductivity. ITO-patterned glass substrates were sonicated in acetone, methanol, and 2-propanol for $15 \mathrm{~min}$ each and later dried in an oven. After that, the ITO substrates were treated with UV-ozone followed by organic layers deposition. NPD, DCDPA, DBFPO:TDBA-DI and TSPO1 were thermally deposited on the substrate in an inert chamber under a pressure of $<4 \times 10^{-4} \mathrm{~Pa}$. The deposition rates of organic layers were $0.1-0.2 \mathrm{~nm} \mathrm{~s}^{-1}$. TQ2f and T2Qf were spin coated from THF solutions at $4000 \mathrm{r}$. p.m. for $45 \mathrm{~s}$. The solution concentration was $10 \mathrm{mg} \mathrm{mL}^{-1}$. The solution concentration and spin rotation were optimized by performing several experiments. The deposition rate of LiF layer was $0.005-0.01 \mathrm{~nm} \mathrm{~s}^{-1}$, whereas for Al layer was $0.3-0.4$ $\mathrm{nm} \mathrm{s}^{-1}$. After the deposition of all layers, the OLED devices were encapsulated with a capping glass in an evaporation chamber filled with nitrogen. The OLED characteristics of all fabricated devices were recorded at RT in an air atmosphere using Agilent $4156 \mathrm{C}$ semiconductor parameter analyzer to monitor the electrical characteristics. The current density-voltage $(J-V)$, luminance-voltage $(L-V)$, and EL spectra were measured using a Konica Minolta CS-100A luminance meter and a CS2000A spectrometer coupled with a Keithley $2635 \mathrm{~A}$ voltage and current source meter. The EQE measurements were conducted using C9920-12, Hamamatsu Photonics, Japan, with a source meter 2400, Keithley, Japan. Time-resolved PL measurements of the EML were conducted using a Hamamatsu streak camera containing a photocathode sensitized from the visible spectrum to $1300 \mathrm{~nm}$ and operated in the synchroscan mode. The samples were excited with $100 \mathrm{fs}$-long optical pulses at $365 \mathrm{~nm}$ by the frequency-doubled output of a mode locked Ti:sapphire laser. The film thickness was measured using a Dektak AlphaStep Profiler.

Photoluminescence measurements. Steady-state PL measurements of TQs, DBFPO, and TSPO1 films were carried out on a Horiba Fluorolog-3 spectrometer (FL 3-22, Horiba Jobin Yvon). Samples were excited at different wavelengths (404 $\mathrm{nm}$ for the TQs and $280 \mathrm{~nm}$ for TSPO1, DBFPO, and mixtures) with a slit width of $10 \mathrm{~nm}$ and the emitted photons were collected in front-face geometry. Timecorrelated single-photon counting measurements were carried out on the DeltaFlex system (Horiba Scientific). For fluorescence measurements, samples were excited with a pulsed laser diode at $404 \mathrm{~nm}$ (NanoLED, pulse width $<200 \mathrm{ps}$ ). For phosphorescence measurements, samples were excited with a SpectraLED at $361 \mathrm{~nm}$. The emitted photons were collected with a picosecond photon detector (PPD-900). The TRPL decay curves of the EML was recorded at a detection wavelength of $450 \mathrm{~nm}$.

Angular-dependent fluorescence. Thin films of DBFPO:TDBA-DI were deposited on pre-cleaned glass substrates by vapor deposition and encapsulate with a capping glass under nitrogen atmosphere. The sample was attached to a hemicylinder prism with matched refractive index oil, and an excitation continuous wave laser with a wavelength of $365 \mathrm{~nm}$ and a power of $<20 \mathrm{~mW}$ was illuminated on the thin films. Through a $410 \mathrm{~nm}$ cut-off filter, a polarizer, and collimating lens, PL intensity in a transverse magnetic mode was detected by a calibrated multichannel spectrometer (PMA-12, Hamamatsu Photonics). The PL intensities were acquired in each out-of-plane angle from $0^{\circ}$ (vertical to the substrate surface) to $90^{\circ}$ (horizontal to the substrate surface) with a step of $2^{\circ}$. The collected radiation patterns were analyzed using a commercially available software (Setfos 4.0, Fluxim AG, Switzerland). The measurements were conducted in an inert nitrogen atmosphere to avoid photochemical degradation as well as PL quenching induced by a combination of oxygen and UV light. The measurements were repeated on different samples to ensure reproducible and consistent results. The dipole orientation of the TADF emitter in the host matrix was determined from the analysis of the angle-dependent PL spectra. The spectra were resolved by using a $p$-polarizing filter and were measured by a fiber optical spectrometer. The angle-dependent $p$ polarized emission intensity at the peak wavelength of the PL spectrum of the emitting layer was detected. The emitting dipole orientation (i.e., the horizontal dipole ratio $\Theta_{/ /}$) was then determined by least square fitting of the measured angledependent $p$-polarized emission intensity with calculated results.

Simulations. The theoretical limit of the device performance was performed using optical simulations based on the plane wave decomposition and as demonstrated by the scattering matrix formalism. Our simulations allowed us to calculate the maximum possible EQE, depending on the film thickness and refractive index in the stack layout by assuming balanced charge carrier injection and a singlet-triplet ratio of unity.

TOF-SIMS measurements. A TOF-SIMS V spectrometer was utilized to depth profile the organic materials and completed devices. Analysis was completed utilizing a three-lens $30 \mathrm{kV}$ BiMn primary-ion gun and the $\mathrm{Bi}^{+}$primary-ion beam (operated in bunched mode, $10 \mathrm{~ns}$ pulse width, analysis current $1.0 \mathrm{pA}$ ) was scanned over a $25 \times 25 \mu \mathrm{m}$ area. Depth profiling was accomplished with a $1 \mathrm{kV}$ oxygen-ion sputter beam $(10.8 \mathrm{nA}$ sputter current) raster of $150 \times 150 \mu \mathrm{m}$. During profiling, all the spectra were collected at or below a primary-ion dose density of $1 \times 10^{12}$ ions $\mathrm{cm}^{-2}$ to remain at the static SIMS limit. The data are plotted with the intensity for each signal at each data point normalized to the total ion counts measured at that data point, which diminishes artifacts from a changing ion yield in different layers when profiling through completed devices.

Gel permeation chromatography. Average molecular weights per number $\left(\bar{M}_{n}\right)$ and polydispersity indices (Đ) were determined with GPC at $80^{\circ} \mathrm{C}$ on a Shimadzu 
liquid chromatograph (LC-20AD) system consisting of a DGU-20A5R degassing unit, a SIL-20AC HT auto sampler, a CTO-20AC column oven, a SPD-20AV UVVis detector, and a RID-20A refractive index detector connected in series. The system contains a PL-GEL $10 \mu \mathrm{m}$ guard column, two PL-GEL $10 \mu \mathrm{m}$ Mixed-C columns, and THF as the eluent. The instrument was calibrated with narrow polystyrene standards with $M_{\mathrm{p}}$ ranging from $4730 \mathrm{~g} \mathrm{~mol}^{-1}$ to $3187000 \mathrm{~g} \mathrm{~mol}^{-1}$.

Nuclear magnetic resonance. ${ }^{1} \mathrm{H}$ - and ${ }^{13} \mathrm{C}$-NMR measurements were carried out in solutions ( $1 \% \mathrm{w} / \mathrm{v}$ ) of the copolymers using $\mathrm{CDCl}_{3}$ (Acros $99.6 \%$ ) as the solvent and tetramethylsilane as the integral standard on a Varian $600 \mathrm{MHz}$ NMR spectrometer at ambient temperature.

Absorption spectrometry. UV-Vis absorption of the polymer solutions (concentration $10^{-5} \mathrm{M}$ ) and the fabricated films were recorded with a Shimadzu 1900 spectrometer.

Cyclic voltammetry. Cyclic voltammetry was conducted on a VersaSTAT4 potentiostat galvanostat with platinum $(\mathrm{Pt})$ disk, $\mathrm{Pt}$ wire, and $\mathrm{Ag} / \mathrm{AgNO}{ }_{3}$ electrode as the working electrode, counter electrode, and reference electrode, respectively, using a $0.1 \mathrm{M}$ solution of tetrabutylammonium hexafluorophosphate $\left(\mathrm{n}-\mathrm{Bu}_{4} \mathrm{NPF}_{6}\right)$ in anhydrous acetonitrile at a potential scan rate of $50 \mathrm{mV} \mathrm{s}^{-1}$. Thin films of samples were deposited onto the Pt disk working electrode from a chloroform solution. The potential of $\mathrm{Ag} / \mathrm{AgNO}_{3}$ reference electrode was internally calibrated by using the ferrocene/ferrocenium redox couple $\left(\mathrm{Fc}_{\mathrm{Fc}}+\mathrm{Fc}^{+}\right.$. The electrochemical energy levels were estimated by using the empirical formula:

$\mathrm{E}_{\mathrm{LUMO}}=-\left[4.80+\left(\mathrm{E}_{\text {onse }}{ }^{\mathrm{Ox}}-\mathrm{Fc}^{1 / 2}\right)\right]$.

Theoretical calculations. All calculations of the model compounds studied in this work have been performed using the Gaussian 09 software package. The alkyl side chains substituents anchored onto the quinoxaline have been replaced with methyl groups in the model compounds for our calculations. Although the presence of these long alkyl chains enhances the solubility of these polymers and affects the charge carrier mobility and photovoltaic behavior of the polymer, from a computational point of view their replacement with shorter chains does not affect their optoelectronic properties (HOMO, LUMO, and bandgap) and thus the optimized structures of the molecules. The ground-state geometry of each model compound has been determined by a full geometry optimization of its structural parameters using the density functional theory (DFT) calculations, upon energy minimization of all possible isomers. In this work, the DFT calculations were performed using the HSEH1PBE/6-311G(d,p) basis set. All calculations were performed taking into account that the system is under vacuum conditions. No symmetry constraints were imposed during the optimization process. The geometry optimizations have been performed with a tight threshold that corresponds to root mean square residual forces smaller than $10^{-5}$ au for the optimal geometry. The energy level of the $\mathrm{E}_{\mathrm{HOMO}}$ and the $\mathrm{E}_{\mathrm{LUMO}}$ of the repetitive units of each polymer were carried out by using the same set of calculations. DFT/HSEH1PBE/6-311G has been found to be an accurate formalism for calculating the structural and electronic properties of many molecular systems. In our studies, the theoretical calculations were performed on dimer model compounds. The visualization of the molecular orbitals has been performed using GaussView 5.0.

\section{Data availability}

The data that support the findings of this study are available on request from the corresponding authors (M.V., A.R.b.M.Y and N.G.)

Received: 24 February 2020; Accepted: 20 July 2021;

Published online: 11 August 2021

\section{References}

1. Di, D. et al. High-performance light-emitting diodes based on carbenemetalamides. Science 356, 159-163 (2017).

2. Cai, X. \& Su, S.-J. J. Marching toward highly efficient, pure-blue and stable thermally activated delayed fluorescent organic light-emitting diodes. Adv. Funct. Mater 28, 1802558 (2018)

3. Lee, J.-H. et al. Blue organic light-emitting diodes: current status, challenges, and future outlook. J. Mater. Chem. C 7, 5874-5888 (2019).

4. Cui, L. S. et al. Long-lived efficient delayed fluorescence organic light-emitting diodes using n-type hosts. Nat. Commun. 8, 2250 (2017).

5. Murawski, C., Leo, K. \& Gather, M. Efficiency roll-off in organic light-emitting diodes. Adv. Mater. 25, 6801-6827 (2013).

6. Adachi, C., Baldo, M. A., Thompson, M. E. \& Forrest, S. R. Nearly $100 \%$ internal phosphorescence efficiency in an organic light emitting device. $J$. Appl. Phys. 90, 5048-5051 (2001).
7. Baldo, M. A., Lamansky, S., Burrows, P. E., Thompson, M. E. \& Forrest, S. R. Very high-efficiency green organic light-emitting devices based on electrophosphorescence. Appl. Phys. Lett. 75, 4-6 (1999).

8. Su, S.-J., Shiba, T., Takeda, T. \& Kido, J. Pyridine-containing triphenylbenzene derivatives with high electron mobility for highly efficient phosphorescent OLEDs. Adv. Mater. 20, 2125-2130 (2008).

9. Lee, C. W. \& Lee, J. Y. Above $30 \%$ external quantum efficiency in blue phosphorescent organic light-emitting diodes using pyrido[2,3- b]indole derivatives as host materials. Adv. Mater. 25, 5450-5454 (2013).

10. Goushi, K., Yoshida, K., Sato, K. \& Adachi, C. Organic light-emitting diodes employing efficient reverse intersystem crossing for triplet-to-singlet state conversion. Nat. Photonics 6, 253-258 (2012).

11. Uoyama, H., Goushi, K., Shizu, K., Nomura, H. \& Adachi, C. Highly efficient organic light-emitting diodes from delayed fluorescence. Nature 492, 234-238 (2012).

12. Tao, Y. et al. Thermally activated delayed fluorescence materials towards the breakthrough of organoelectronics. Adv. Mater. 26, 7931-7958 (2014).

13. Lin, T. A. et al. Sky-blue organic light emitting diode with $37 \%$ external quantum efficiency using thermally activated delayed fluorescence from spiro acridine-triazine hybrid. Adv. Mater. 28, 6976-6983 (2016).

14. $\mathrm{Wu}, \mathrm{T}$.-L. et al. Diboron compound-based organic light-emitting diodes with high efficiency and reduced efficiency roll-off. Nat. Photonics 12, 235-240 (2018).

15. Kondo, Y. et al. Narrowband deep-blue organic light-emitting diode featuring an organoboron-based emitter. Nat. Photonics 13, 678-682 (2019).

16. Nguyen, T. B., Nakanotani, H., Hatakeyama, T. \& Adachi, C. The role of reverse intersystem crossing using a TADF-type acceptor molecule on the device stability of exciplex-based organic light-emitting diodes. Adv. Mater. 32, 1906614 (2020).

17. Zhang, Q. et al. Design of efficient thermally activated delayed fluorescence materials for pure blue organic light emitting diodes. J. Am. Chem. Soc. 134, 14706-14709 (2012)

18. Zhang, Q. et al. Efficient blue organic light-emitting diodes employing thermally activated delayed fluorescence. Nat. Photonics 8, 326-332 (2014).

19. Ahn, D. H. et al. Highly efficient blue thermally activated delayed fluorescence emitters based on symmetrical and rigid oxygen-bridged boron acceptors. Nat. Photonics 13, 540-546 (2019).

20. Li, W. et al. Spiral donor design strategy for blue thermally activated delayed fluorescence emitters. ACS Appl. Mater. Interfaces 13, 5302-5311 (2021)

21. Liu, Y., Li, C., Ren, Z., Yan, S. \& Bryce, M. R. All-organic thermally activated delayed fluorescence materials for organic light-emitting diodes. Nat. Rev. Mater. 3, 18020 (2018).

22. Seino, Y., Sasabe, H., Pu, Y.-J. \& Kido, J. High-performance blue phosphorescent OLEDs using energy transfer from exciplex. Adv. Mater. 26, 1612-1616 (2014)

23. Bencheikh, F., Sandanayaka, A. S. D., Fukunaga, T., Matsushima, T. \& Adachi, C. Origin of external quantum efficiency roll-off in 4,40-bis[(N-carbazole) styryl]biphenyl (BSBCz)-based inverted organic light emitting diode under high pulsed electrical excitation. J. Appl. Phys. 126, 185501 (2019).

24. Khramtchenkov, D. V., Bässler, H. \& Arkhipov, V. I. Charge carrier recombination in organic bilayer electroluminescent diodes. I. Theory. J. Appl. Phys. 81, 6954-6962 (1997).

25. Giebink, N. \& Forrest, S. R. Quantum efficiency roll-off at high brightness in fluorescent and phosphorescent organic light emitting diodes. Phys. Rev. B 77, 235215 (2008)

26. Kotadiya, N. B., Blom, P. W. M. \& Wetzelaer, G.-J. A. H. Efficient and stable single-layer organic light emitting diodes based on thermally activated delayed fluorescence. Nat. Photonics 13, 765-769 (2019).

27. Kotadiya, N. B. et al. Universal strategy for Ohmic hole injection into organic semiconductors with high ionization energies. Nature 17, 329-334 (2018).

28. Lee, J. et al. Deep blue phosphorescent organic light-emitting diodes with very high brightness and efficiency. Nat. Mater. 15, 92-99 (2016).

29. Singh, R. et al. The impact of thienothiophene isomeric structures on the optoelectronic properties and photovoltaic performance in quinoxaline based donor-acceptor copolymers. Polym. Chem. 6, 3098-3109 (2015).

30. Verstappen, P. et al. Fluorination as an effective tool to increase the opencircuit voltage and charge carrier mobility of organic solar cells based on poly (cyclopenta[2,1-b:3,4-b'] dithiophene-alt-quinoxaline) copolymers. J. Mater. Chem. A 3, 2960-2970 (2015).

31. Yamamoto, K. et al. Effects of fluorine substitution in quinoidal oligothiophenes for use as organic semiconductors. J. Mater. Chem. C. 8, 3580-3588 (2020).

32. Luo, H. et al. Remarkable enhancement of charge carrier mobility of conjugated polymer field-effect transistors upon incorporating an ionic additive. Sci. Adv. 2, e1600076 (2016)

33. Sarma, M. \& Wong, K.-T. Exciplex: an intermolecular charge-transfer approach for TADF. ACS Appl. Mater. Interfaces 10, 19279-1930 (2018).

34. Colella, M., Pander, P., de Sa Pereira, D. \& Monkman, A. P. Interfacial TADF exciplex as a tool to localize excitons, improve efficiency, and increase OLED lifetime. ACS Appl. Mater. Interfaces 10, 40001-40007 (2018). 
35. Vecchi, P. A., Padmaperuma, A. B., Qiao, H., Sapochak, L. S. \& Burrows, P. E. A dibenzofuran-based host material for blue electrophosphorescence. Org. Lett. 8, 4211-4214 (2006).

36. Jeon, S. O., Jang, S. E., Son, H. S. \& Lee, J. Y. External quantum efficiency above $20 \%$ in deep blue phosphorescent organic light-emitting diodes. Adv. Mater. 23, 1436-1441 (2011).

37. Song, W., Lee, H. L. \& Lee, J. Y. High triplet energy exciplex hosts for deep blue phosphorescent organic light-emitting diodes. J. Mater. Chem. 5, 5923-5929 (2017).

38. dos Santos, P. L., Dias, F. B. \& Monkman, A. P. Investigation of the mechanisms giving rise to TADF in exciplex states. J. Phys. Chem. C 120 18259-18267 (2016)

39. Xiang, C., Peng, C., Chen, Y. \& Franky So, F. Origin of sub-bandgap electroluminescence in organic light-emitting diodes. Small 11, 5439-5443 (2015).

40. Song, J. et al. Lens free OLEDs with over $50 \%$ external quantum efficiency via external scattering and horizontally oriented emitters. Nat. Commun. 9, 3207 (2018).

41. Kim, S.-Y. et al. Organic light-emitting diodes with $30 \%$ external quantum efficiency based on a horizontally oriented emitter. Adv. Funct. Mater. 23, 3896-3900 (2013).

42. Wang, Q. \& Aziz, H. Degradation of organic/organic interfaces in organic light-emitting devices due to polaron-exciton interactions. ACS Appl. Mater. Interfaces 5, 8733-8739 (2013).

43. Rai, D. \& Holmes, R. J. Measurement of the triplet exciton diffusion length in organic semiconductors. J. Mater. Chem. C 7, 5695-5701 (2019).

44. Sandanayaka, A. S. D., Matsushima, T. \& Adachi, C. Degradation mechanisms of organic light-emitting diodes based on thermally activated delayed fluorescence molecules. J. Phys. Chem. C 119, 23845-23851 (2015).

45. Jeltsch, K. F., Lupa, G. \& Weitz, R. T. Materials depth distribution and degradation of a FIrpic based solution-processed blue OLED. Organ. Electron. 26, 365-370 (2015).

46. Aizawa, N. et al. Solution-processed multi-layer small-molecule light-emitting devices with high-efficiency white-light emission. Nat. Commun. 5, 5756 (2014).

47. Jørgensen, M., Norrman, K. \& Krebs, F. C. Stability/degradation of polymer solar cells. Sol. Energy Mater. Sol. Cells 92, 686-714 (2008).

48. Kim, J. U. et al. Nanosecond-time-scale delayed fluorescence molecule for deep-blue OLEDs with small efficiency roll off. Nat. Commun. 11, 1765 (2020).

49. Hirata, S. et al. Highly efficient blue electroluminescence based on thermally activated delayed fluorescence. Nat. Mater. 14, 330-336 (2015).

50. Zimmermann, D. et al. Synthesis of D- $\pi-A-\pi$ type benzodithiophenequinoxaline copolymers by direct arylation and their application in organic solar cells. J. Polym. Sci. A Polym. Chem. 56, 1457-1467 (2018).

\section{Acknowledgements}

M.V. acknowledges support of this work by the project "Development of Materials and Devices for Industrial, Health, Environmental and Cultural Applications” (MIS
5002567), which is implemented under the "Action for the Strategic Development on the Research and Technological Sector," funded by the Operational Programme "Competitiveness, Entrepreneurship and Innovation" (NSRF 2014-2020) and co-financed by Greece and the European Union (European Regional Development Fund). J.J. acknowledges the National Research Foundation of Korea (NRF), grant No (2020M3H4A1A02084899).

\section{Author contributions}

M.V., M.D., N.G., A.R.b.M.Y., W.J.d.S., A.S., J.C., A.E.X.G., A.G.M., G.P. and F.K.S. performed the device experiments. G.R. and G.C.V. performed the synthesis of DBFPO. C.L.C. performed the design and synthesis of copolymers. N.G., M.D., P.J. and. Y.D. performed the photoluminescence measurements and analysis. M.V., A.R.b.M.Y., N.G., J.J. and J.S.K. developed the idea, supervised all experiments, and contributed to data analysis. M.V. and N.G. wrote the paper with contribution from all authors.

\section{Competing interests}

The authors declare no competing interests.

\section{Additional information}

Supplementary information The online version contains supplementary material available at https://doi.org/10.1038/s41467-021-25135-z.

Correspondence and requests for materials should be addressed to M.V., Abd.R.b.M.Y., C.L.C. or N.G.

Peer review information Nature Communications thanks the anonymous reviewer(s) for their contribution to the peer review of this work.

Reprints and permission information is available at http://www.nature.com/reprints

Publisher's note Springer Nature remains neutral with regard to jurisdictional claims in published maps and institutional affiliations.

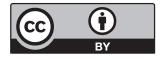

Open Access This article is licensed under a Creative Commons Attribution 4.0 International License, which permits use, sharing, adaptation, distribution and reproduction in any medium or format, as long as you give appropriate credit to the original author(s) and the source, provide a link to the Creative Commons license, and indicate if changes were made. The images or other third party material in this article are included in the article's Creative Commons license, unless indicated otherwise in a credit line to the material. If material is not included in the article's Creative Commons license and your intended use is not permitted by statutory regulation or exceeds the permitted use, you will need to obtain permission directly from the copyright holder. To view a copy of this license, visit http://creativecommons.org/ licenses/by/4.0/

(C) The Author(s) 2021 